\title{
Importância da Ultra-Sonografia Anorretal Tridimensional na Decisão Terapêutica da Endometriose Profunda
}

\author{
Importance of the Three-Dimensional Anorectal Ultrasonography \\ in Deep Endometriosis
}

\begin{abstract}
UNIVALDO ETSUO SAGAE ${ }^{1}$; DORYANE MARIADOS REIS LIMA²; NAMIR CAVALLI ${ }^{3}$; LUCIAMATIKO TAKAMATSU SAGAE $^{4}$; TOMAZ MASSAYUKI TANAKA ${ }^{5}$; MAURO WILLEMANN BONATTO ${ }^{6}$; RICARDO SHIGUEO TSUCHIYA ${ }^{7}$; CARLOS ALBERTO DE CARVALHO ${ }^{8}$; ANDREA ISHIKAWA SHIRATORI ${ }^{9}$
\end{abstract}

\begin{abstract}
${ }^{1}$ Mestre em Cirurgia do Aparelho Digestivo - USP. Professor da Disciplina de Gastroenterologia e Cirurgia do Aparelho Digestivo da UNIOESTE - Universidade Estadual do Oeste do Paraná; ${ }^{2}$ Mestre em Cirurgia - UFC/CE. Professora Convidada da Disciplina de Introdução à Clínica, Técnica Cirúrgica e Anestesiologia da UNIOESTE - Universidade Estadual do Oeste do Paraná; ${ }^{3}$ Presidente da SOBENGE. Chefe do Departamento de Ginecologia e Obstetrícia da UNIOESTE - Universidade Estadual do Oeste do Paraná; ${ }^{4}$ Especialista em Ginecologia e Obstetrícia; ${ }^{5}$ Doutor em Cirurgia - UNIFESP/Escola Paulista de Medicina. Professor da Disciplina de Introdução à Clínica, Técnica Cirúrgica e Anestesiologia da UNIOESTE -

Universidade Estadual do Oeste do Paraná; ${ }^{6}$ Mestre em Clínica Cirúrgica-UFPR. Coordenador da Disciplina de Gastroenterologia e Cirurgia do Aparelho Digestivo da Universidade Estadual do Oeste do Paraná; ${ }^{7}$ Orofessor da Disciplina de Introdução à Clínica, Técnica Cirúrgica e Anestesiologia da UNIOESTE - Universidade Estadual do Oeste do Paraná; ${ }^{8}$ Mestre em Cirurgia - UNIFESP/Escola Paulista de Medicina; ${ }^{9}$ Especialista em Radiologia.
\end{abstract}

SAGAE UE; LIMADMR; CAVALLI N; SAGAE LMT; TANAKA TM; BONATTO MW; TSUCHIYA; CARVALHO CA; SHIRATORI AI. Importância da Ultra-Sonografia Anorretal Tridimensional na Decisão Terapêutica da Endometriose Profunda. Rev bras Coloproct, 2009;29(4): 435-442.

RESUMO: OBJETIVO: Este estudo visa demonstrar a importância da ultra-sonografia anorretal tridimensional (US 3D) no diagnóstico da endometriose profunda e o grau de acometimento do trato intestinal na decisão terapêutica da endometriose do septo retovaginal. MÉTODOS: Estudo prospectivo realizado entre março de 2007 e julho de 2009. Sessenta e cinco mulheres com endometriose pélvica e com queixas gastrointestinais foram avaliadas e submetidas a US 3D. Vinte pacientes, média de idade 33,7anos, com suspeita de foco endometriótico intestinal foram submetidas ao procedimento laparoscópico para a realização de inventário da cavidade abdominal e tratamento cirúrgico. RESULTADOS: Em dezenove mulheres (95\%), os achados laparoscópicos confirmaram a presença do foco endometriótico retal. $O$ procedimento realizado à laparoscopia foi: exérese de foco peritoneais $(n=1)$; ressecção parcial do retossigmóide $(n=9)$; exérese de nódulo de reto $(n=10)$. $O$ tempo operatório médio por procedimento foi de 120 minutos. O tempo médio de alta foi 1,7 dias. Duas pacientes apresentaram como complicação o aparecimento de fistula retovaginal. CONCLUSÃO: Conclui-se que a ultra-sonografia anorretal tridimensional é exame específico na avaliação do segmento anorretal, decisivo na detecção de focos endometrióticos do septo retovaginal e avalia eventuais doenças associadas nesse segmento, determinando a estratégica terapêutico-cirúrgica adequada.

Descritores: Endometriose, Ultra-sonografia, Cirurgia Colorretal Diagnóstico, Laparoscopia.

Trabalho realizado na Universidade Estadual do Oeste do Paraná - Centro Médico Hospitalar Genesis - Gastroclínica Cascavel Ltda.

Recebido em 17/09/2009

Aceito para publicação em 18/11/2009 
Rev bras Coloproct Outubro/Dezembro, 2009
Importância da Ultra-Sonografia Anorretal Tridimensional na Decisão Terapêutica da Endometriose Profunda Univaldo Etsuo Sagae e Cols.
Vol. 29 $\mathbf{N}^{\circ} 4$

\section{INTRODUÇÃO}

Endometriose é uma afecção freqüente em mulheres na idade reprodutiva com incidência de 10\%$20 \%{ }^{(1)}$. É causa de infertilidade em $25 \%-50 \%$ dessas pacientes $^{(2)}$. A localização mais freqüente são os ovários e superfície peritoneal, seguido das localizações profundas, isto é, que penetram o espaço subperitoneal mais do que $5 \mathrm{~mm}^{(3)}$, sendo mais acometidos os ligamentos uterosacrais, fundo de saco retovaginal, reto, sigmóide e septo retovaginal ${ }^{(4)}$.

Na década de 1990, o estudo da endometriose foi alvo de modificações estratégicas a partir de sua crescente prevalência e complexidade que envolve seu diagnóstico e tratamento. A partir da descrição em 1992 da classificação da endometriose conforme sua profundidade $^{(5)}$ e da classificação em doenças distintas localizadas em ovário, peritônio e septo retovaginal ${ }^{(6)}$, atenção específica tem sido dada aos aspectos diagnósticos e terapêuticos da moléstia envolvendo esses três sítios.

As lesões da endometriose profunda diferenciam-se da superficial por apresentarem comportamento agressivo e por não responderem aos mecanismos de defesa do fluido peritoneal ${ }^{(7)}$. São lesões caracterizadas por serem, muitas vezes, refratárias a tratamento medicamentoso e o principal tratamento nesses casos é a ressecção cirúrgica das lesões ${ }^{(8)}$.

A laparoscopia é o exame gold standard para diagnóstico e avaliação da severidade da endometriose peritoneal segundo revisão publicada pela American Fertility Society Score (AFS, 1985). Porém, as lesões têm avaliação dificultada pela laparoscopia devido à característica em iceberg ${ }^{(9)}$. Raramente há o acometimento da mucosa, portanto, os métodos diagnósticos por via anorretais habitualmente utilizados são pouco elucidativos.

A modalidade de ultra-sonografia anorretal tridimensional (US 3-D) é capaz de diagnosticar infiltração da parede retal e áreas adjacentes corretamente, nos casos de endometriose pélvica profunda. É útil na determinação da escolha da técnica cirúrgica, pois permite medir a distância do foco endometriótico ao aparelho esfincteriano, bem como seu tamanho, grau de infiltração na parede retal e sua distância aos esfíncteres anais ${ }^{(10)}$, estabelecendo qual o procedimento cirúrgico mais adequado para cada caso. Por estas razões, a infiltração da parede retal deveria ser diagnosticada antes da cirurgia.

\section{OBJETIVO}

Este estudo visa demonstrar a importância da ultra-sonografia anorretal tridimensional no diagnóstico da endometriose profunda e o grau de acometimento do trato intestinal na decisão terapêutica da endometriose do septo retovaginal.

\section{METODOLOGIA}

Estudo prospectivo realizado entre março de 2007 e julho de 2009. Sessenta e cinco mulheres com endometriose pélvica e queixas gastrointestinais foram encaminhadas ao Serviço de Ginecologia do Hospital Universitário de Cascavel (UNIOESTE) e Centro Médico Hospitalar Genesis, avaliadas e submetidas a US 3-D pelo Serviço de Coloproctologia do Hospital Universitário e Gastroclínica de Cascavel.

Trinta e nove mulheres, com média de idade de 33,7 anos (23-43) anos, apresentaram suspeita de foco endometriótico na parede retal. Vinte destas pacientes foram submetidas ao procedimento laparoscópico para retirada das lesões e associado à Corrida dos Órgãos Peritoneais na Endometriose $(\mathrm{COPE})^{(11)}$ para a realização de inventário da cavidade abdominal. Esse grupo de mulheres foi inclusa no presente estudo. O critério de exclusão foi: pacientes que não foram submetidas à laparoscopia.

\section{RESULTADOS}

Trinta e nove pacientes apresentaram imagem sugestiva de endometriose intestinal a US 3D, sendo que 20 foram submetidas ao procedimento laparoscópico, onde há 4 trocarteres conforme figura 1. Quinze $(75 \%)$ mulheres apresentavam-se com foco na gordura perirretal (figura 2), duas (10\%) na camada muscular do reto (figura 3) e três $(15 \%)$ em todas as camadas do reto (figura 4). Em dezenove, os achados laparoscópicos confirmaram a presença do foco endometriótico retal como sugerido pela US 3D. Ao inventário da cavidade, foram identificados outros focos: no sigmóide (figura 5) $(\mathrm{n}=3-15 \%)$, no apêndice cecal (figura 6) $(\mathrm{n}=4-20 \%)$, no peritônio de fundo de saco $(n=3-15 \%)$ e diafragma $(n=1-$ $5 \%$ ). Houve confirmação histológica dos mesmos (figura 7).

O procedimento realizado à laparoscopia foi: lise de bridas intraperitoneais exérese de foco 
Univaldo Etsuo Sagae e Cols.

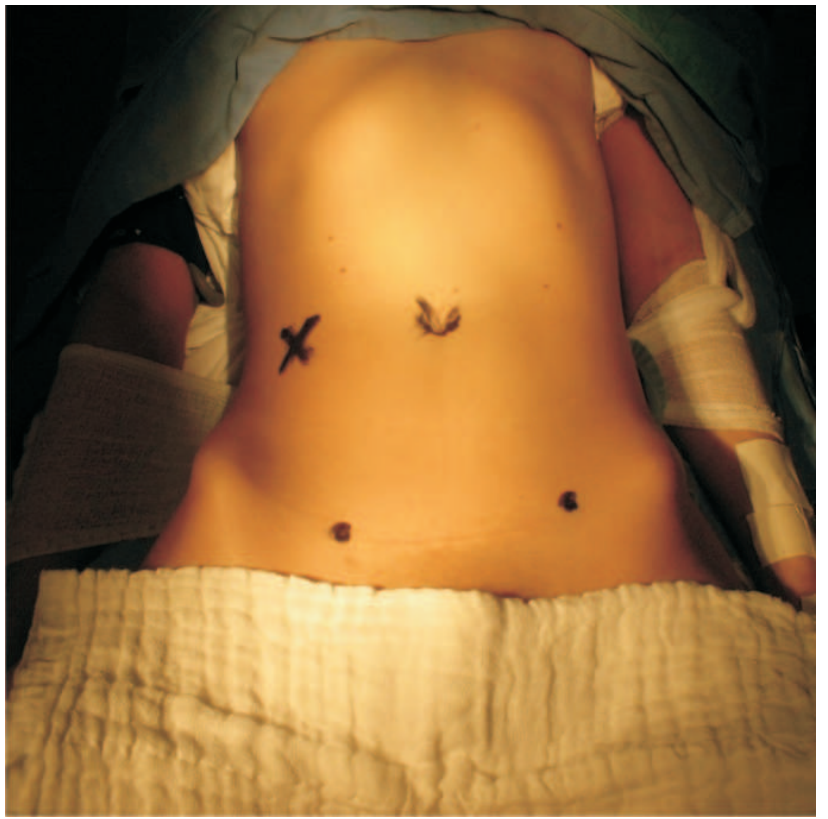

Figura 1 - Locais de inserção dos trocarteres durante laparoscopia.

peritoneais $(\mathrm{n}=1-5 \%)$; ressecção parcial do retossigmóide $(\mathrm{n}=9-45 \%)$; exérese de nódulo de reto $(\mathrm{n}=10-50 \%)$. Como procedimentos associados podese citar: apendicectomia $(\mathrm{n}=4-20 \%)$, exérese de nódulo de epiplon $(n=1-5 \%)$, exérese de nódulos de peritônio $(n=5-25 \%)$ e exérese de nódulo de diafrag- ma $(\mathrm{n}=1-5 \%)$. O tempo operatório médio por procedimento foi de 120 minutos (60 a 300 minutos). O tempo médio de alta foi 1,7 dias (1 a 3 dias).

Duas pacientes apresentaram como complicação o aparecimento de fistula retovaginal. Uma havia sido submetida à exérese da lesão e outra a retossigmoidectomia com exteriorização da peça pela vagina. O quadro clínico de ambas apresentou-se no oitavo dia de pós-operatório.

A cirurgia foi necessária na primeira paciente, pois apresentava sinais de peritonite. $\mathrm{O}$ tratamento foi conservador com medicação constipante na outra paciente, pois a fístula foi caracterizada de baixo débito. Ambas evoluíram bem. Nesta série não houve casos de sangramento volumoso (definido como necessidade de reintervenção).

\section{DISCUSSÃO}

Estima-se que 3-37\% de todas as pacientes com endometriose apresentem comprometimento intestinal, característica esta presente em até $50 \%$ das endometrioses severas ${ }^{(12)}$. Aproximadamente $42 \%$ têm comprometimento intestinal ${ }^{(13)}$. O segmento mais acometido é o retossigmóide, com incidência de $72 \%$, seguido pelo íleo terminal em $14 \%$, reto em $14 \%$, ceco e

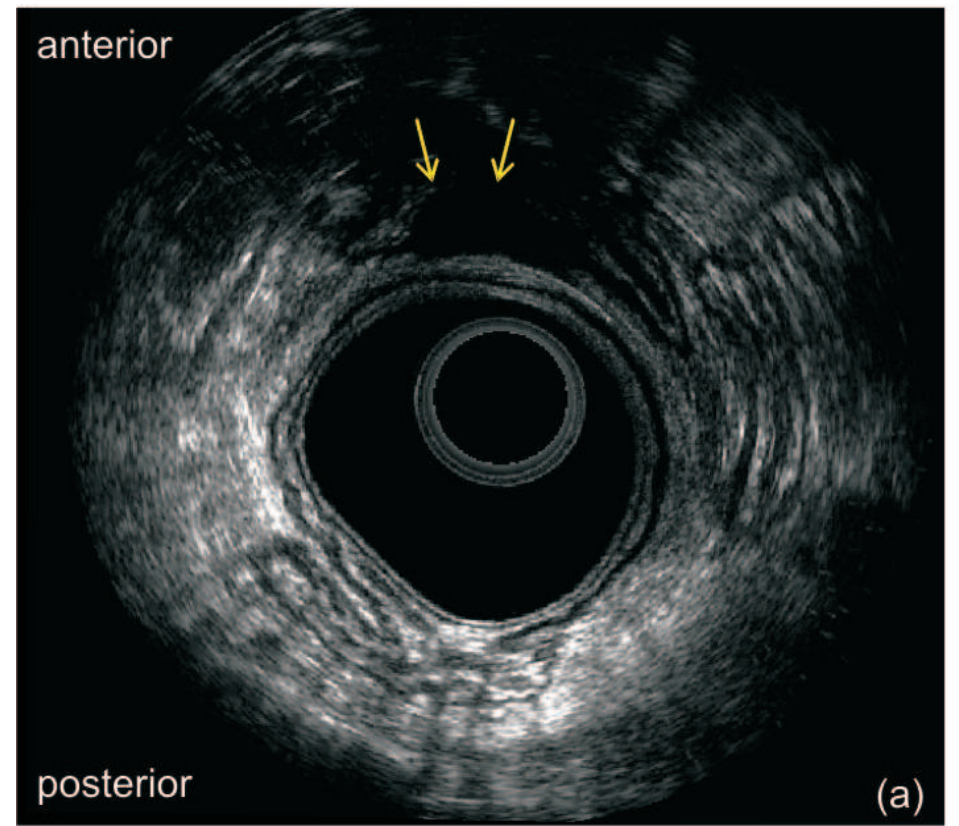

Figura 2 - infiltração do foco na gordura perirretal. a-corte axial: lesão presente no quadrante anterior do reto (setas). b-corte sagital: comprimento longitudinal da lesão (linha).

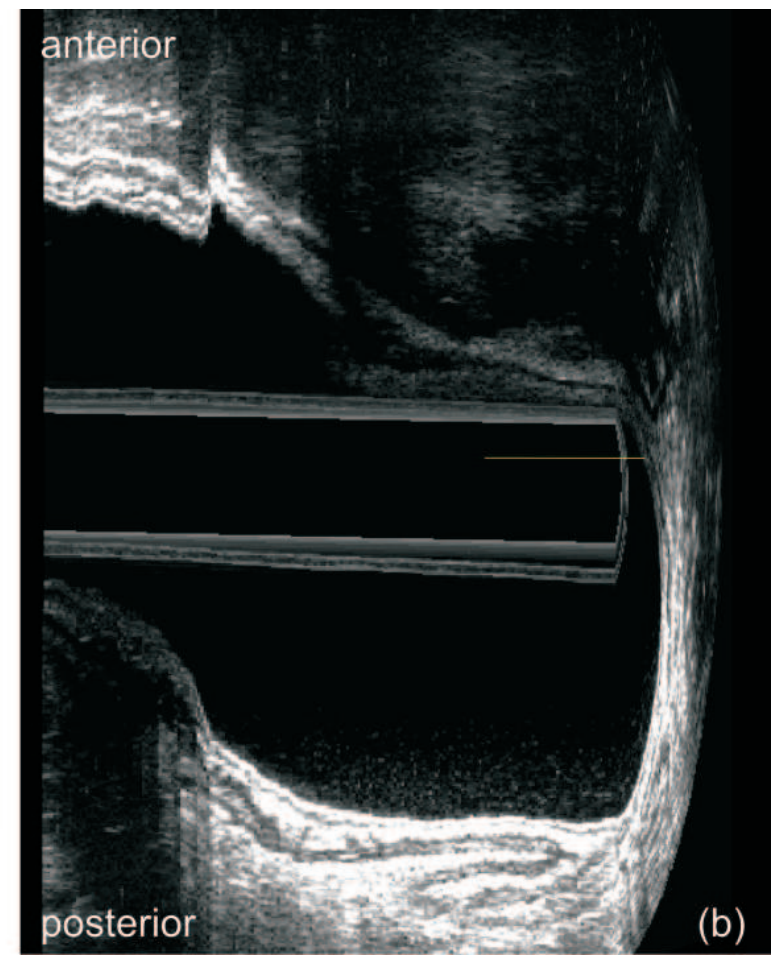




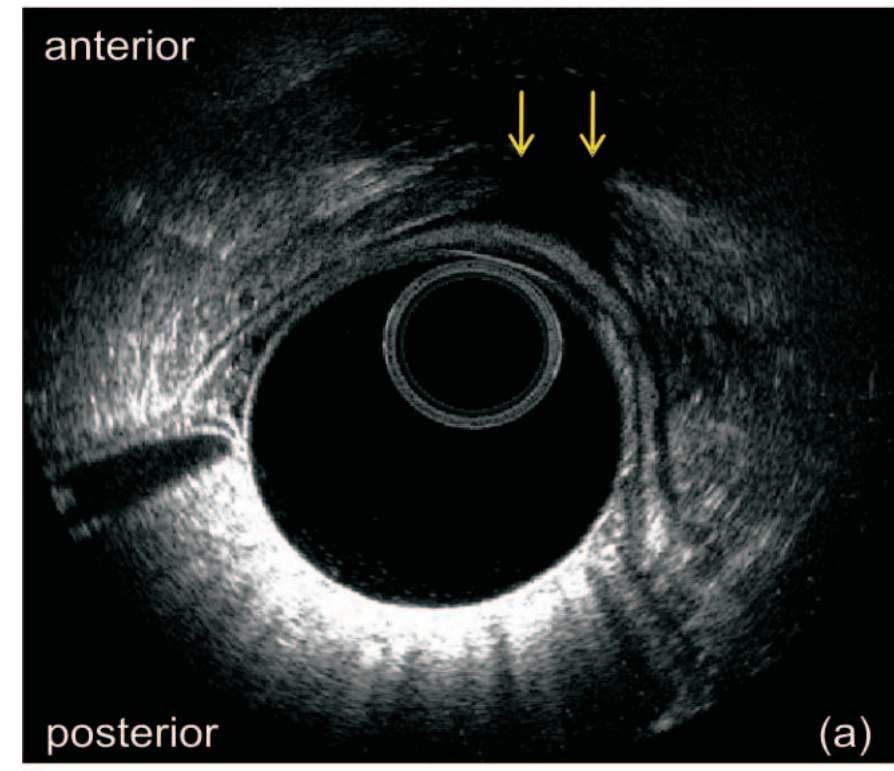

Figura 3 - infiltração do foco na camada muscular do reto. a - corte axial : lesão presente no quadrante anterior do reto (setas). $b$-corte sagital: comprimento longitudinal da lesão (setas).

demais segmentos do cólon, 4\%. É essencial suspeitar de envolvimento intestinal ao lidar com pacientes portadoras de endometriose e adotar condutas pré-operatórias que permitam cirurgias adequadas.

A ultra-sonografia transvaginal (TVUS) é método recomendado para diagnosticar endometriose ovariana e pélvica ${ }^{(14,15,16,17)}$, bem como de bexiga ${ }^{(18)}$. A TVUS é tão eficiente quanto à ressonância magnética nuclear para o diagnóstico de endometriose pélvica anterior e ovariana ${ }^{(19)}$. Mas é menos efetiva em diagnosticar a endometriose pélvica posterior porque não

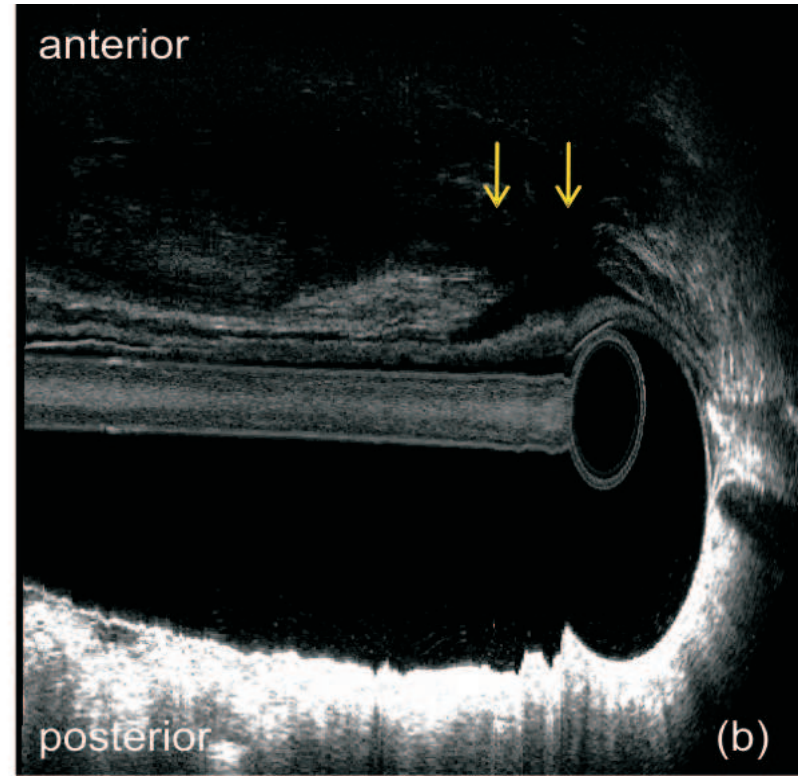

avalia com precisão a infiltração das camadas da parede retal ${ }^{(20,21,22)}$. Em serviços não referenciados, esse exame passa a ser menos eficaz, pois não é realizado preparo intestinal adequado e o examinador não é habilitado na evidenciação dos focos na parede retal e área adjacente ${ }^{(23)}$. Essa característica é considerada uma limitação do método.

A modalidade de ultra-sonografia anorretal propõe diagnosticar a endometriose pélvica posterior, em especial se há acometimento do septo retovaginal. Consiste em uma técnica simples, não
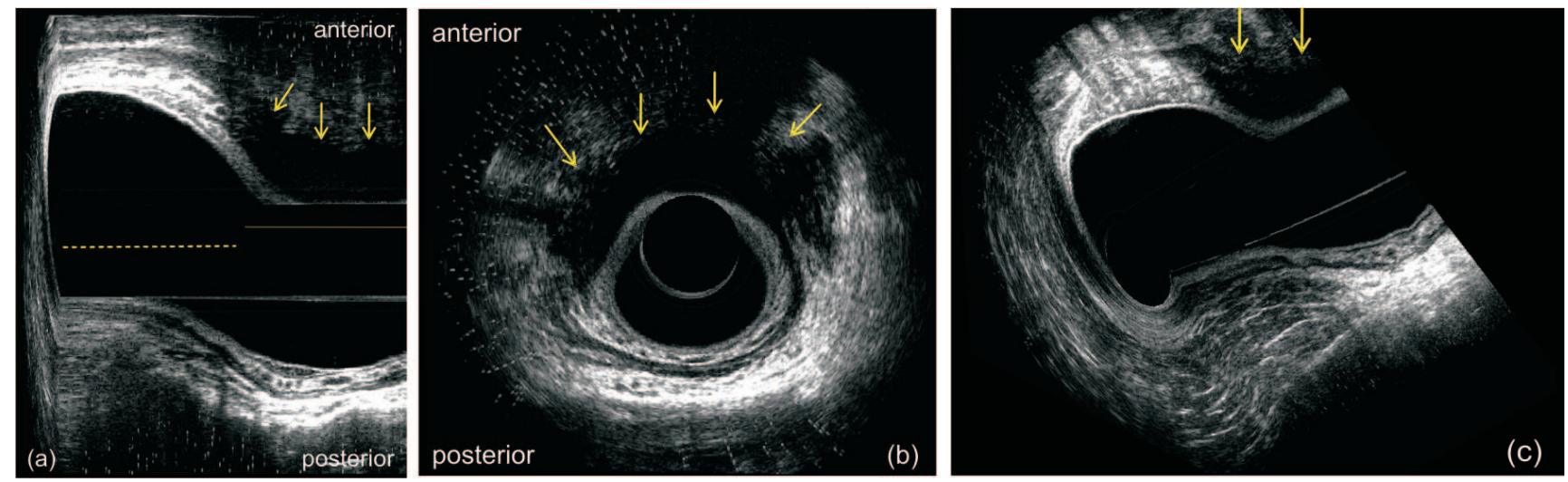

Figura 4 - infiltração do foco em todas as camadas do reto.

a-corte sagital: foco invadindo até mucosa retal (setas).

$b$ - corte axial: foco invadindo 50\% da circunferência retal (setas).

$c$-corte transversal (setas). 
Univaldo Etsuo Sagae e Cols.
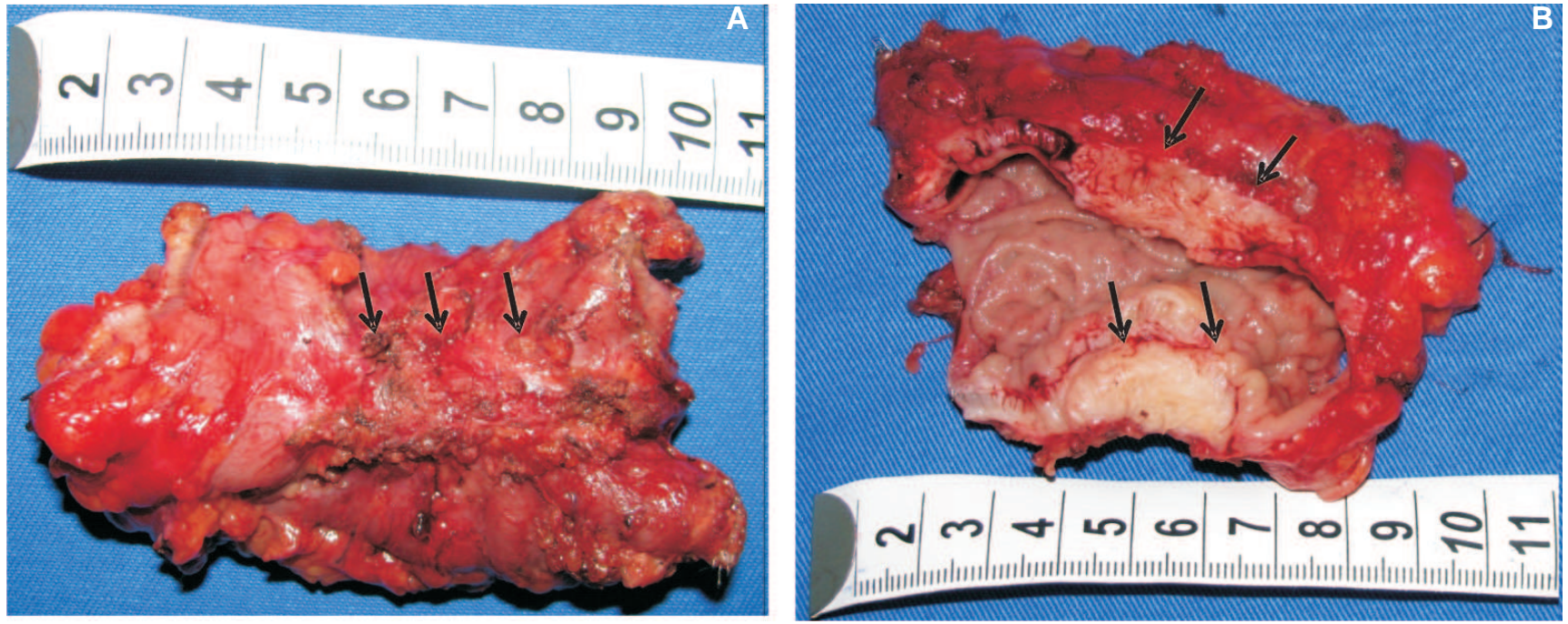

Figura 5 - infiltração do sigmóide (peça cirúrgica).

a - peça fechada: lesão com retração da parede do segmento intestinal (setas).

$b$ - peça aberta: evidenciação da lesão comprometendo todas as camadas do segmento intestinal (setas).
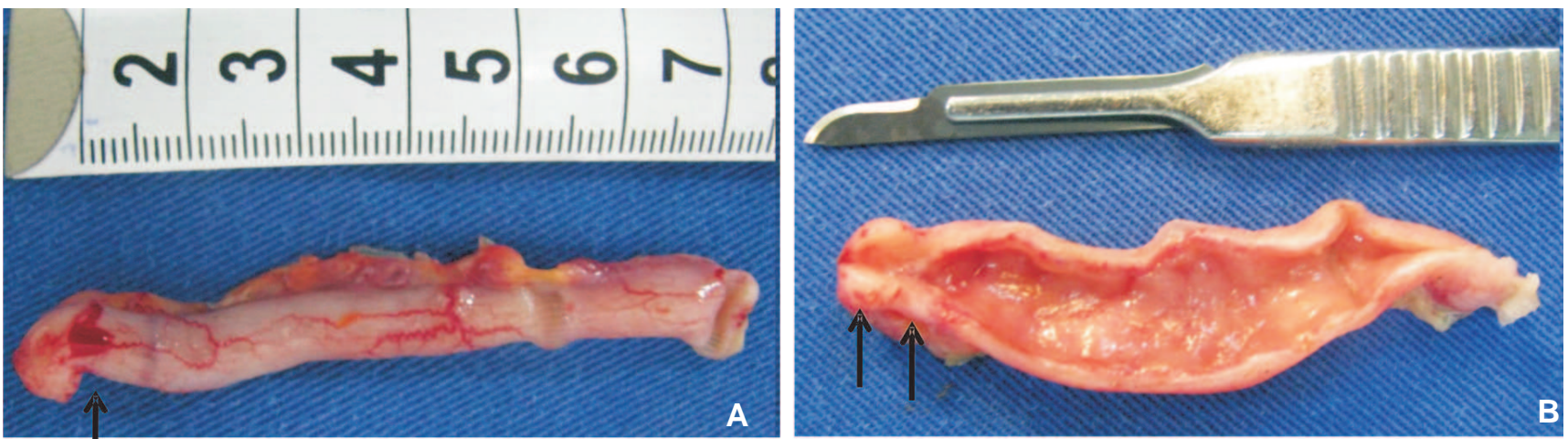

Figura 6 - infiltração do apêndice cecal (peça cirúrgica).

a - peça fechada: lesão com retração da ponta do apêndice (setas).

b-peça aberta: evidenciação da lesão comprometendo todas as camadas do segmento (setas).

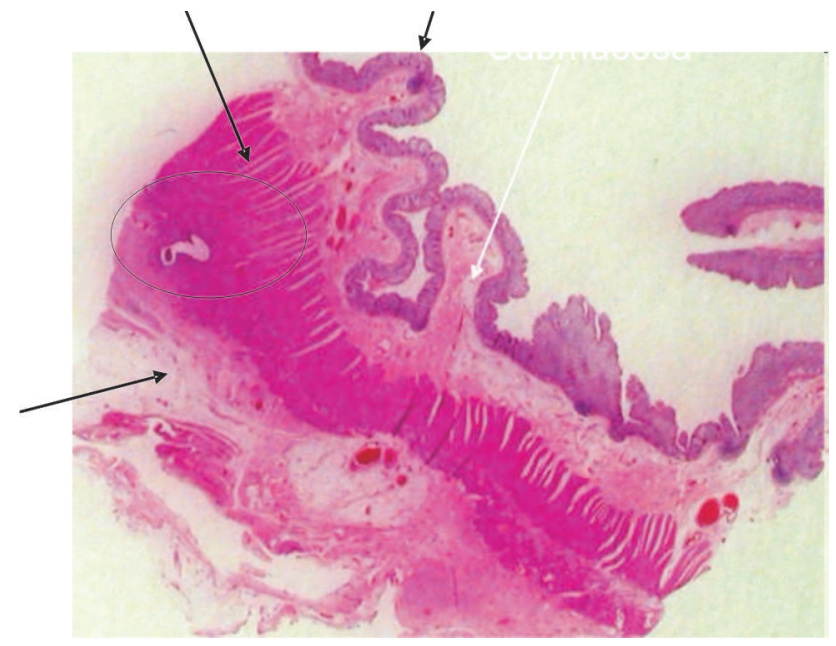

Figura 7 - Vista panorâmica mostrando corte de intestino com foco com endometriose afetando partes profundas de túnicas musculares próprias (círculo). invasiva e capaz de diagnosticar infiltração da parede retal e áreas adjacentes. É útil na determinação da escolha da técnica cirúrgica, pois permite medir a distância do foco endometriótico ao aparelho esfincteriano, estabelecendo qual o procedimento cirúrgico mais adequado para cada caso. Por estas razões, a infiltração da parede retal deve ser diagnosticada antes da cirurgia. Muitos autores têm demonstrado a boa sensibilidade e especificidade do exame, numa percentagem de 87,5 e 97 , respectivamente, para o diagnóstico de infiltração da parede retal por focos endometrióticos ${ }^{(19)}$.

As imagens visualizadas pela ultra-sonografia sugestivas de focos de endometriose caracterizam-se como áreas hipoecóicas, heterogêneas, localizadas na gordura perirretal ou infiltrando as camadas da parede 
retal. Apresentam-se com maior diâmetro fora da parede do reto, ou seja, infiltrando as camadas da parede retal de fora para dentro, em geral, até a muscular própria, mas podendo acometer as demais camadas até a mucosa. Porém, a superfície da mucosa é raramente danificada.

A proposta deste estudo é ressaltar a importância do transdutor anorretal tridimensional na avaliação pélvica posterior em pacientes com endometriose. A característica deste transdutor é permitir a reconstrução tridimensional, após as imagens serem captadas no modo bi-dimensional, possibilitando avaliar as lesões em múltiplos planos e medir com precisão o comprimento longitudinal e a distância para os músculos esfincterianos ${ }^{(10)}$, (figura 8) provendo, assim, informações indispensáveis para a escolha da abordagem terapêutico-cirúrgica.

Outra vantagem a ser considerada é que esta modalidade de exame permite, também, avaliar outras patologias do ânus e reto associadas, tais como hemorróidas, rotura esfincteriana, retocele,

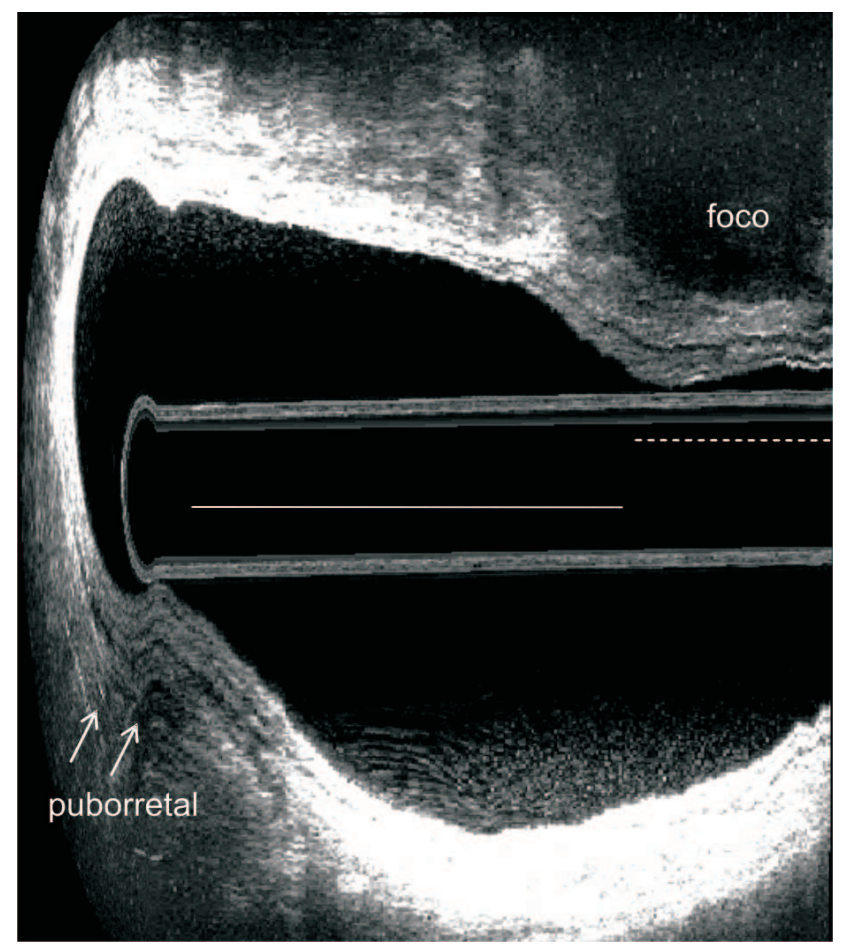

Figura 8- corte sagital do reto inferior: foco invadindo gordura perirretal - medição do comprimento longitudinal (linha pontilhada) e a distância desta para os músculos esfincterianos (linha contínua). dentre outras alterações orificiais que, eventualmente, possam interferir no resultado final da cirurgia proposta primariamente. $\mathrm{O}$ cirurgião, assim, fica de posse de todas as informações importantes da região anorretal, associada à investigação da endometriose profunda.

Neste estudo, das 20 pacientes que foram submetidas ao procedimento laparoscópico, houve confirmação dos achados ultrasonográficos em dezenove $(95 \%)$ dos casos. Nessa única paciente que apresentou achados ultrasonográficos de comprometimento retal, mas não foi confirmado pela laparoscopia, apenas apresentava-se com focos peritoneais e no apêndice cecal. Com isso, percebese a importância dessa modalidade de exame para orientação pré-operatória nas pacientes portadoras de endometriose profunda. Os autores ressaltam que como a característica dessas lesões é em iceberg há dificuldade de avaliação pela laparoscopia, já que elas não são facilmente evidenciadas no peritônio. Em tendo um exame com grau de sensibilidade apresentada, pode-se ter um planejamento cirúrgico da equipe médica e preparo adequado da paciente. A equipe médica terá á disposição o material cirúrgico adequado para aperfeiçoar o procedimento e evitar complicações e a paciente será informada das possibilidades cirúrgicas bem como da eventual necessidade de ostomia protetora. Pois se deve lembrar que essa é uma afecção benigna e que acomete mulheres jovens, como demonstra esse estudo onde a média de idade das pacientes foi de 33,7 anos. Muitas delas ainda apresentam desejo de fertilidade e preocupação estética.

A evolução dos métodos de imagem proporcionou uma contribuição importante, quantitativamente e qualitativamente no diagnóstico e, conseqüentemente, na abordagem terapêutica mais adequada. Com isso, há uma tendência a que estes exames se incorporem na rotina pré-operatória.

\section{CONCLUSÃO}

Conclui-se que a ultra-sonografia anorretal tridimensional é exame específico na avaliação do segmento anorretal, decisivo na detecção de focos endometrióticos do septo retovaginal e avalia eventuais doenças associadas nesse segmento, determinando a estratégica terapêutico-cirúrgica adequada. 
Rev bras Coloproct

Outubro/Dezembro, 2009
Importância da Ultra-Sonografia Anorretal Tridimensional na Decisão Terapêtica da Endometriose Profunda

Univaldo Etsuo Sagae e Cols.
Vol. 29

ABSTRACT: OBJECTIVE: This study aims to demonstrate the importance of three-dimensional anorectal ultrasonography (US 3D) in the diagnosis of deep endometriosis and level of intestinal involvement in the decision of the therapy of endometriosis of rectovaginal septum. METHODS: A prospective study between March 2007 and July 2009. Sixty-five women with pelvic endometriosis and gastrointestinal complaints were evaluated and submitted to 3D U.S.. Twenty patients, mean age 33.7 years, with suspected of intestinal endometriosis were submitted to laparoscopic procedure and surgical treatment. RESULTS: In nineteen women $(95 \%)$, the laparoscopic findings confirmed endometriosis in the rectum. The procedure was performed to laparoscopy: the focus peritoneal excision $(n=1)$, partial resection of rectosigmoid $(n=9)$, excision of a nodule of rectum $(n=10)$. The average operative time per procedure was 120 minutes. The average time to stay in hospital was 1.7 days. Two patients had the appearance as complication of rectovaginal fistula. CONCLUSION: We conclude that three-dimensional anorectal ultrasonography is specific in the assessment of anorectal segment, crucial for detecting endometriosis focus of the rectovaginal septum and evaluates possible associated diseases in this segment, determining the appropriate therapeutic strategy and surgical.

Key words: Endometriosis, Ultrasonography, Colorectal Surgery, Diagnosis, Laparoscopy.

\section{REFERÊNCIAS}

1. Bis K G, Vrachliotis T G, Agrawal R, Shetty A N, Maximovich A, Hricak H. Pelvic endometriosis: MR imaging spectrum with laparoscopic correlation and diagnostic pitfalls. Radiographics 1997; 17:639-55.

2. Imaoka I, Wada A, Matsuo M, Yoshida M, Kitagaki H, Sugimura K. MR Imaging of disorders associated with female infertility: use in diagnosis, treatment, and management. Radiographics 2003; 23:1401-21.

3. Koninckx P R, Meuleman C, Demeyere S, et al. Suggestive evidence that pelvic endometriosis is a progressive disease, whereas deeply infiltrating endometriosis is associated with pelvic pain. Fertil Steril 1991; 55:759-65.

4. Chapron C, Dubuisson JB. Management of deep endometriosis. Ann N Y Acad Sci 2001; 943:276-80.

5. Koninckx P R, Martin D C. Deep endometriosis: a consequence of infiltration or retraction or possibly adenomyosis externa? Fertil Steril 1992; 58:924-8.

6. Nisolle M, Donnez J. Peritoneal endometriosis, ovarian endometriosis, and adenomyotic nodules of the rectovaginal septum are three different entities. Fertil Steril 1997; 68:58596.

7. Dmowski WP. Etiology and histogenesis of endometriosis. Ann N Y Acad Sci. 1991;622:236-41.

8. Graham B, Mazier WP. Diagnosis and management of endometriosis of the colon and rectum. Dis Colon Rectum. 1988; 31(12):952-6.

9. Koninckx PR, Riittinen L, Seppala M, Cornillie FJ CA-125 and placental protein 14 concentrations in plasma and peritoneal fluid of women with deeply infiltrating pelvic endometriosis. Fertil Steril. 1992 Mar;57(3):523-30.

10. Murad-Regadas S M, Regadas F S P. Ultra-sonografia anorretal bi e tridimensional In: distúrbios funcionais do assoalho pélvico. Atlas de Ultra-sonografia Anorretal Bi e Tridimensional. Rio de Janeiro: Revinter; 2006.
11. Sagae E U, Lopasso F, Abrão M S, Cavalli N, Rodrigues J J G. Endometriose do trato gastrintestinal - correlações clínicas e laparoscópicas. Rev bras de coloprocto 2007; 27(4): 423-31.

12. Macaffe $\mathrm{C} \mathrm{H}$, Greer H L. Intestinal endometriosis. A report of 29 cases and a survey of the literature. J Obstet Gynaecol. 1960; 67:539-55.

13. Piketty M, Chopin N, Dousset B, Millischer-Bellaische AE, Roseau G, Leconte M, et al. Preoperative work-up for patients with deeply infiltrating endometriosis: transvaginal ultrasonography must definitely be the first-line imaging examination. C.Hum Reprod. 2009; 24(3):602-7.

14. Guerriero S, Mais V, Ajossa S, Paoletti A M, Angiolucci M, Labate $\mathrm{F}$, et al.The role of endovaginal ultrasound in differentiating endometriomas from other ovarian cysts. Clin. Exp. Obstet. Gynecol.1995; 22(1):20-2.

15. Friedman H, Vogelzang R L, Mendelson E B, Neiman H L, Cohen M. Endometriosis detection by US with laparoscopic correlation. Radiology. 1985; 157 (1): 217-20.

16. Dessole S, Farina M, Rubattu G, Cosmi E, Ambrosini G, Nardelli G B. Sonovaginography is a new technique for assessing rectovaginal endometriosis. Fertil Steril 2003; 79:1023-7.

17. Bazot M, Detchev R, Cortez A, Amouyal P, Uzan S, Darai E. Transvaginal sonography and rectal endoscopic sonography for the assessment of pelvic endometriosis: a preliminary comparison. Hum Reprod 2003; 18:1686-92.

18. Fedele L, Bianchi S, Raffaelli R, Portugese A. Pre-operative assessment of bladder endometriosis. Hum. Reprod. 1997; 12(11):2519-22.

19. Bahr A, de Parades V, Gadonneix P, Etienney I, Salet-Lizée D, Villet R, Atienza P.Endorectal ultrasonography in predicting rectal wall infiltration in patients with deep pelvic endometriosis: a modern tool for an ancient disease.Dis Colon Rectum. 2006; 49(6):869-75.

20. Kinkel K, Chapron C, Balleyguier C, Fritel X, Dubuisson J B, Moreau J F. Magnetic resonance imaging characteristics of deep endometriosis. Hum Reprod 1999; 14:1080-6. 
21. Chapron C, Vieira M, Chopin N. Accuracy of rectal endoscopic ultrasonography and magnetic resonance imaging in the diagnosis of rectal involvement for patients presenting with deeply infiltrating endometriosis. Ultrasound Obstet Gynecol 2004; 24:175-9.

22. Camagna O, Dhainaut C, Dupuis O. Surgical management of rectovaginal septum endometriosis from a continuous series of 50 cases. Gynecol Obstet Fertil 2004; 32:199-209.

23. Goncalves MO, Dias JA Jr, Podgaec S, Averbach M, Abrão MS Transvaginal ultrasound for diagnosis of deeply infiltrating endometriosis. Int J Gynaecol Obstet. 2009 Feb;104(2):156-60.

Endereço para correspondência: UNIVALDO ETSUO SAGAE

Endereço: Rua Antonio Alves Massaneiro, 414

Centro - Cascavel - Paraná

Telefone: 4521017700

Fax: 4521017729

Email: sagae@gastro.com.br 